\title{
Study of residual stresses in bimetallic work rolls
}

\author{
Ingrid NEIRA TORRES ${ }^{1,2, a^{*}}$, Gaëtan GILLES ${ }^{1, b}$, \\ Jérôme TCHOUFANG TCHUINDJANG ${ }^{3, c}$, \\ Jacqueline LECOMTE-BECKERS ${ }^{3, \mathrm{~d}}$, Mario SINNAEVE ${ }^{4, \mathrm{e}}$, \\ Anne Marie HABRAKEN ${ }^{1, f}$ \\ ${ }^{1}$ Department ArGEnCo, Division $\mathrm{MS}^{2} \mathrm{~F}$, Université de Liège, Belgium \\ ${ }^{2}$ Department of Materials Engineering, Universidad de Concepción, Chile \\ ${ }^{3}$ Department AME, Division MMS, Université de Liège, Belgium \\ ${ }^{4}$ Marichal Ketin, Verte Voie 39B- 4000 Liège, Belgium

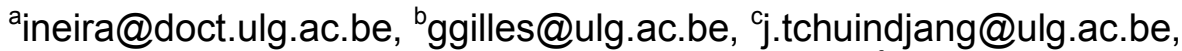 \\ jacqueline.Lecomte@ulg.ac.be, êmario.Sinnaeve@mkb.be, ${ }^{f}$ anne.Habraken@ulg.ac.be
}

Keywords: phase transformation, residual stresses, FE simulations, bimetallic rolls, cooling, heat treatment.

\begin{abstract}
An experimental campaign of compression tests, differential thermal analysis (DTA), differential scanning calorimetry (DSC), dilatometry and microstructure analysis has been performed, as well as the identification of the material data set for finite element (FE) analysis of bimetallic rolls. This article numerically investigates the stress and strain fields after the cooling stage and it checks their effect on the subsequent heat treatment step. As bimetallic rolls have a different material for core and shell, the effect of the roll size and the shell thickness on residual stresses is also studied.
\end{abstract}

\section{Introduction}

Bimetallic rolls are used at the roughing stands of the Hot Strip Mill. These rolls must achieved two basic mechanical properties which are opposite to each other; a high wear resistance in the shell material (High Chrome Steel standing for HCS) and a significant toughness in the core material (Spheroidal Graphite Iron or SGI). The studied bimetallic rolls are manufactured by Marichal Ketin industry (MK) through continuous vertical spin casting process where the HCS shell material is poured into the mould until solidification is completed prior to the pouring of the SGI core material. MK industry has observed several failure cases of bimetallic HCS-SGI rolls for diameters from $1200 \mathrm{~mm}$ during their cooling or heat treatment. FEM simulations are performed using Thermo Mechanical Metallurgical code [1] to analyse the stresses and strains due to the phase transformations and the presence of two different materials during the preliminary cooling stage that follows the casting process, hereinafter referred to as Post Casting Cooling stage (PCC). The subsequent Tempering Heat Treatment (THT) is also simulated. In this step we assume no phase transformation to happen but the difference that exists between Coefficients of Thermal Expansion (CTE) of both materials may generate additional stress and strain fields. A first analysis was presented in a previous work [2] where the cooling of the PCC was simulated. However in the present work, more accurate parameters such as CTE, transformation strains and TTT diagrams are used as input data. The objective of the present work is to simulate the PCC and the subsequent THT of bimetallic rolls considering the most accurate parameters as input data and to study the effect of three different roll sizes in order to understand the fracture event of bigger rolls.

\section{Input Data}

TTT diagram. For both materials, TTT diagrams illustrated at Fig. 1 were obtained using inverse method through FE code using a CCT diagram as input data [3]. In relation to previous work [2], an updated TTT diagram was introduced for core material considering the SGI pearlitic giving the higher failure rate for roll manufacturing. Using this TTT diagram and the temperature history, the 
FE simulation generates a core structure containing $70 \%$ of Pearlite and $30 \%$ of Ferrite. For HCS grade, TTT diagram used allows obtaining a shell structure containing $50 \%$ of retained Austenite and $50 \%$ of Martensite. This phase distribution is in good agreement with the quantitative analysis of phases performed.

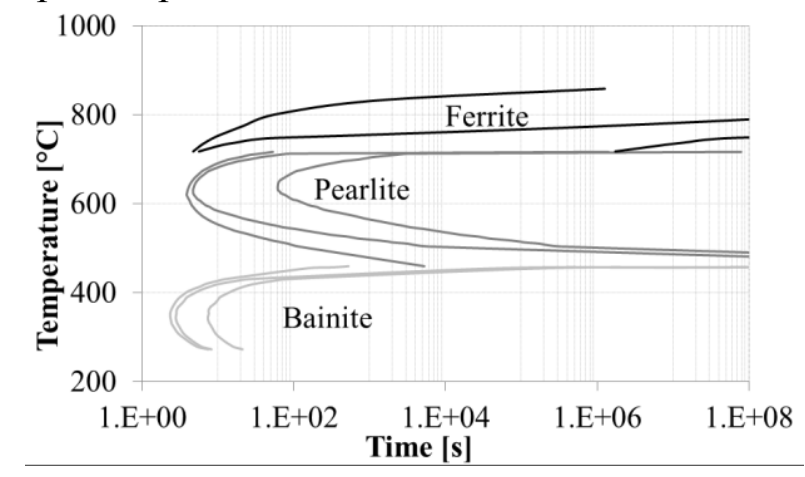

(a)

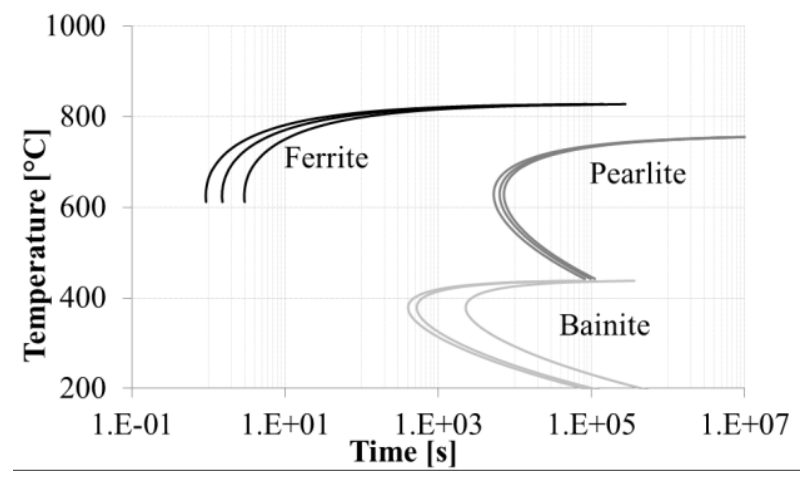

(b)

Fig. 1: TTT diagram used for simulations (a) SGI pearlitic core material (b) HCS shell material.

Coefficient of Thermal Expansion (CTE). Thermo physical data for both materials; SGI and HCS have been experimentally obtained [4],[5]. In particular, dilatometry tests were performed at a constant strain rate of $5^{\circ} \mathrm{C} / \mathrm{min}$ for cooling and heating whose results are presented in Fig. 2. From these experimental curves, it is possible to compute classical metallurgist CTE using Eq. 1 (see results in Fig. 3). Resultant incremental CTE for non linear FE code $\alpha_{\mathrm{FE}}$ for each case are computed and correspond to the parameters considered for cooling and heating simulations.

$$
\alpha_{\text {metallurgist }}(\mathrm{T})=\frac{\frac{\Delta \mathrm{L}(\mathrm{T})}{\mathrm{L}_{0}}}{\mathrm{~T}-\mathrm{T}_{0}}
$$

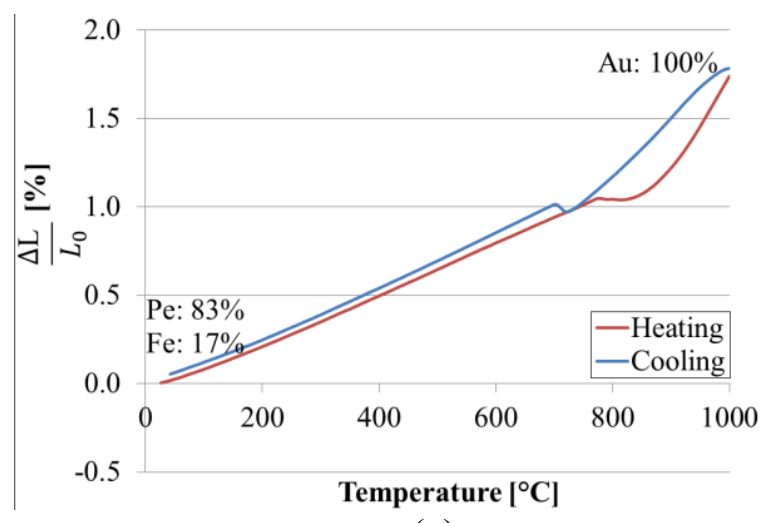

(a)

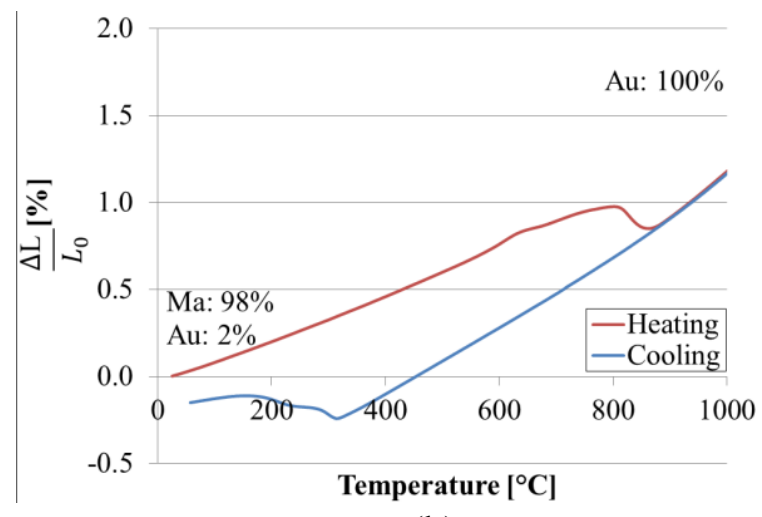

(b)

Fig. 2: Experimental dilatometry curves (a)SGI material (b)HCS material.

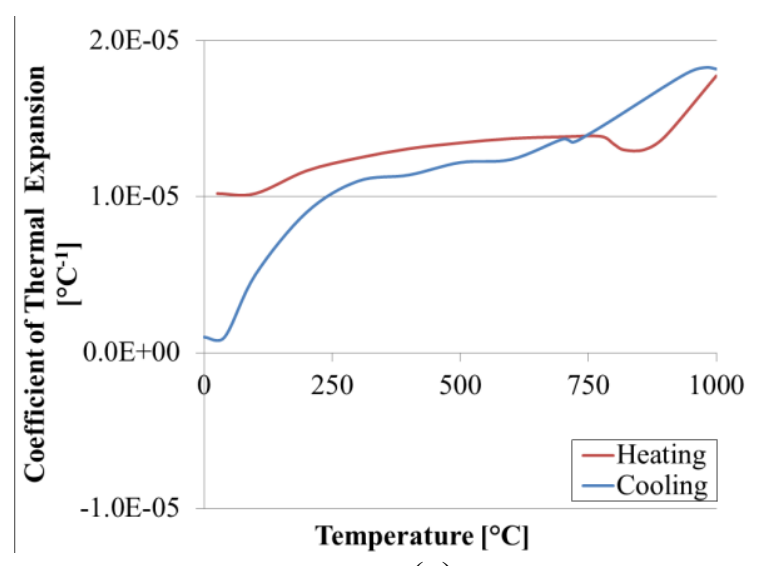

(a)

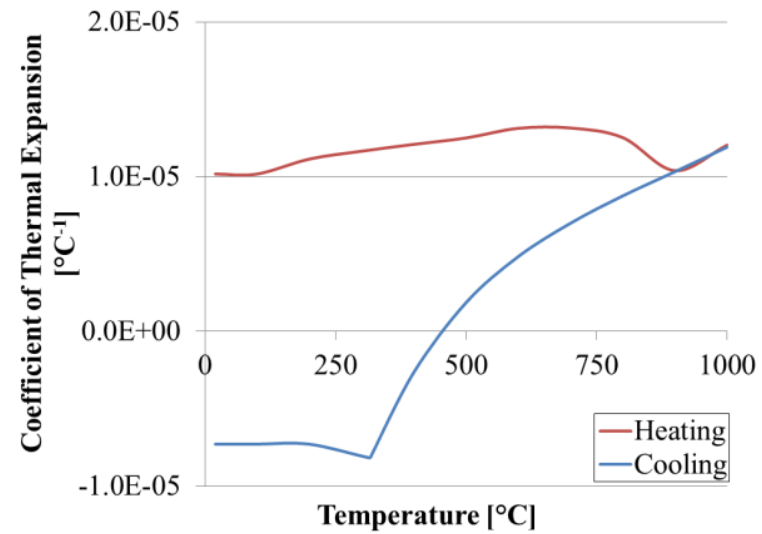

(b)

Fig. 3: Metallurgical CTE including phase transformation (a)SGI material (b)HCS material. 
Transformation strain $\boldsymbol{\varepsilon}^{\mathbf{t r}}$. For each phase transformation, a change of mass density is involved generating stress and strains within the rolls. The value of this parameter is specific for each material and each transformation undergone. In this work, transformation strains for simulated materials were obtained by inverse modelling applied to the dilatometry test. Within the classical phase transformation model used in the code [1], mixture law is used to define dilatation coefficient of multiphase material and transformation strain is related to predicted phase transformation rate based on Koistinen-Marburger [6] law for martensitic transformation and Johnson Mehl Avrami [7] approach for diffusive transformation. The objective of the FE inverse modelling was to identify the correct value of $\varepsilon^{\text {tr }}$ in order to reproduce the cooling dilatometry curve provided by Fig. 2. This parameter defines the peak at the moment of phase transformation. Several trials were performed and by using the parameters given at Table 1, the TTT diagram of Fig. 1, the martensitic transformation start temperature of $313^{\circ} \mathrm{C}$ and the thermo physical data of [4] and [5], it was possible to precisely reproduce the peaks appearing on the dilatometry curves for each material during the cooling sequence. For SGI material, the same value of transformation strain was considered for ferritic and pearlitic transformation. For HCS material, only martensitic transformation was allowed.

Table 1: Transformation strain values.

\begin{tabular}{|l|l|}
\hline Material & $\boldsymbol{\varepsilon}^{\mathbf{t r}}$ \\
\hline SGI (Ferritic and pearlitic transformation) & $7.76 \times 10^{-04}$ \\
\hline HCS (Martensitic transformation) & $3.00 \times 10^{-03}$ \\
\hline
\end{tabular}

\section{Post Casting Cooling (PCC) and subsequent Tempering Heat Treatment (THT) simulations}

Geometry for simulation. Simulated axisymmetric geometry containing 77 elements is illustrated in Fig. 4, where axial stresses and phase evolutions will be studied while focusing of four critical elements. The red element (1) corresponds to the centre of the roll, dark blue element (77) represents the surface and elements yellow (38) and light blue (39) are symbolizing the interface between both core and shell material respectively. For simulations, free dilatation is allowed through the Y axis while spring elements keep parallel edges. The cooling step PCC is modelled through convection radiation; while tempering THT is defined by impose temperature at the surface.

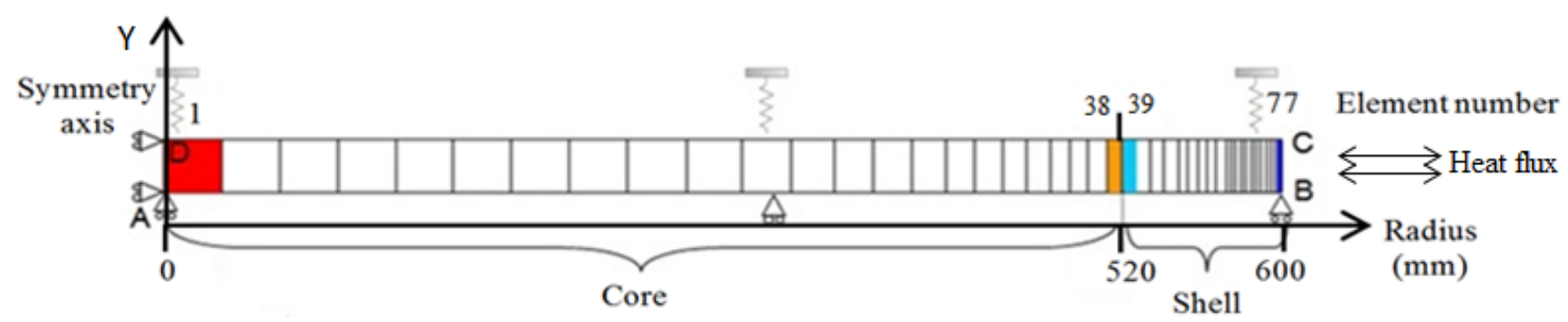

Fig. 4: Geometry for bimetallic roll axisymmetric simulations.

Temperature evolution. After the vertical spin casting, bimetallic rolls are subjected to PCC and THT. Simulations of the complete thermal cycle were performed as failure can happen at different stage of the process. The actual PCC followed by THT cycle performed in a roll in industrial conditions can be described as follows: starting at $1000^{\circ} \mathrm{C}$, the cooling step until room temperature in still air takes around 11 days before launching the tempering stage up to $500^{\circ} \mathrm{C}$ during $40 \mathrm{hr}$. The whole thermal cycles for cylinder centre (1) and surface (77) are drawn in Fig. 5(a), while the predicted thermal gradients along the radius at different times are shown in Fig. 5(b). One can see in continuous lines, the temperature gradients corresponding to the PCC stage and in dashed lines the gradients corresponding to THT stage. Through these figures, it is possible to observe that during cooling the centre of cylinder is at higher temperature than the surface and the contrary effect occurs for heating stage. 


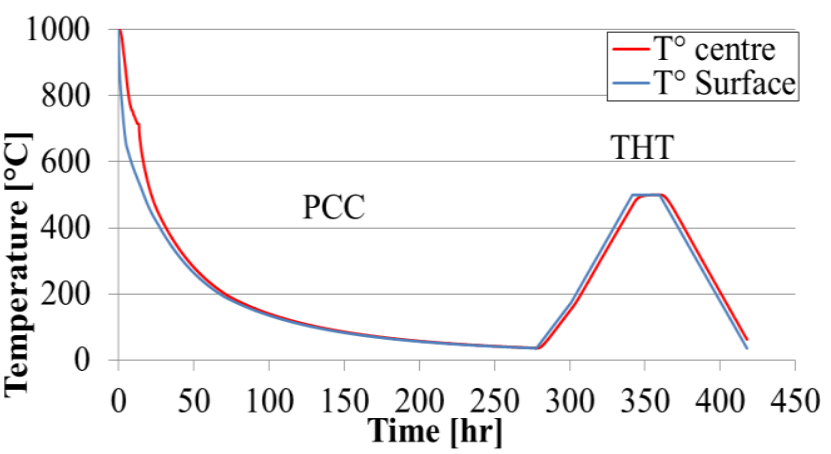

(a)

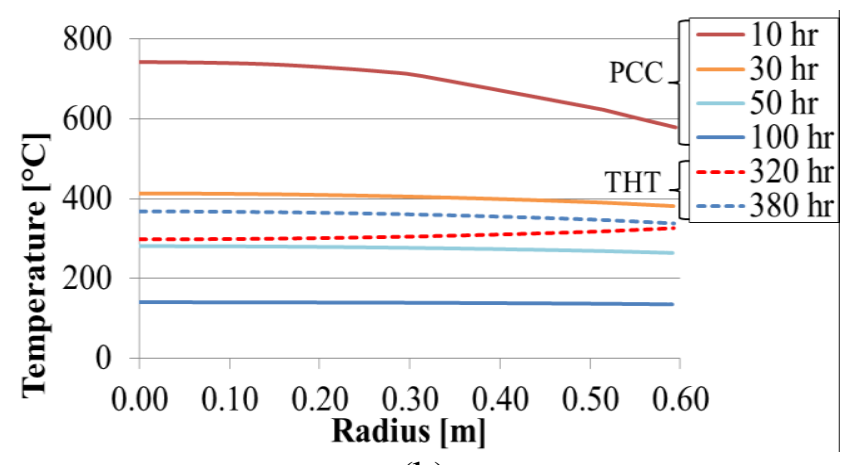

(b)

Fig. 5: (a) Complete thermal cycle simulated for surface (77) and centre (1) (b) Temperature gradients along the radius for different times of the thermal cycle.

Axial stress and phase transformation evolution. Fig. 6(a) illustrates the axial stress evolution during the whole thermal cycle of the simulation for the four elements identified in Fig. 4. For PCC stage, it is observed that important stresses are generated. However it can be noticed that the most important axial stresses are produced during the heat treatment, generating a high tension state in the shell material and a compression state in the core material. It is also important to notice the large difference between the stress states for both interface elements at around $320 \mathrm{hr}$ during heating stage of THT; while element 39 is at $+434 \mathrm{MPa}$, the element 38 is at $-484 \mathrm{MPa}$. A similar effect happens at around $380 \mathrm{hr}$ during cooling stage of THT. Even if FE model is "simplified" while assuming no pollution of the shell material by the core material, the stress gradient is critical in this interface.

A zoom of axial stresses evolution and their interactions with corresponding phase transformations during PCC stage is presented in Fig. 6(b).

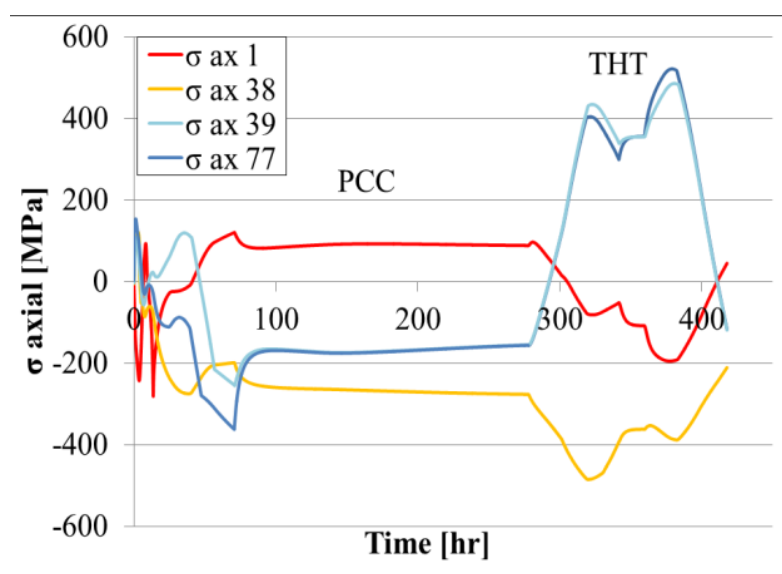

(a)

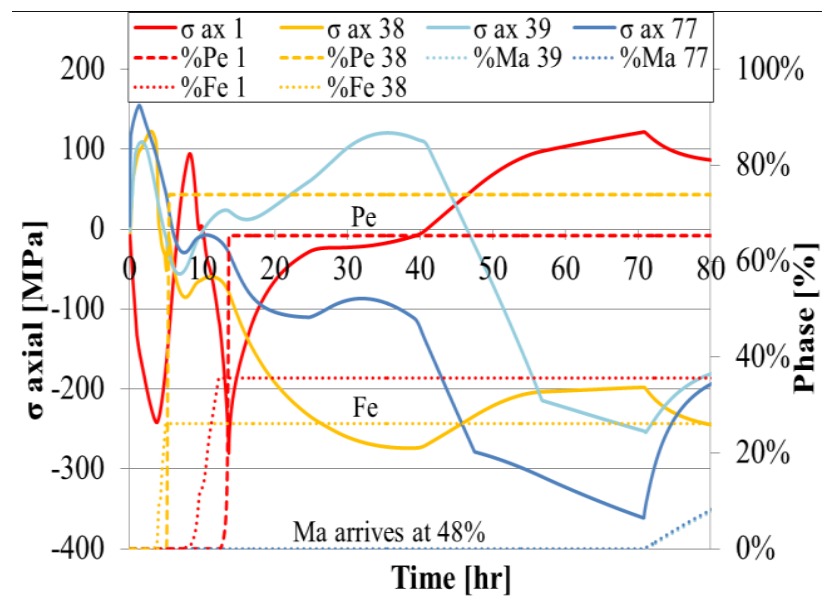

(b)

Fig. 6: Axial stress evolution (a) Complete thermal cycle PCC + THT (b) First $80 \mathrm{hr}$ for PCC $(1=$ center, $38=$ interface core, $39=$ interface shell, $77=$ surface).

During the first $4 \mathrm{hr}$, the shell material is in tension state and the centre of cylinder in compression state due to the thermal gradient. The first phase transformations happen at element (38); ferritic transformation starts at $2.8 \mathrm{hr}$ and pearlitic transformation starts at $4.9 \mathrm{hr}$ generating a compression state in this element (38) and also in shell material. At the same time by equilibrium, the remainder core material not yet transformed (1) goes to tension state until its own phase transformation occurring at $6.7 \mathrm{hr}$ and $12 \mathrm{hr}$ for ferritic and pearlitic transformation respectively. This total core transformation induces a high compression state for this element (1) while the remaining material undergoes tension state by equilibrium.

At around $30 \mathrm{hr}$ the core that reaches a temperature of $400^{\circ} \mathrm{C}$ is completely transformed and the shell is subjected to compression state due to the fact that CTE considerably decrease (becomes negative) at this temperature where core dilatation is still positive (see Fig. 3). The core material goes toward tension as response to the shell material compression leading to a high tension state in centre and low compression state in the core interface. 
For shell material, all the elements are transformed to martensite phase at the same time starting at $71 \mathrm{hr}$ and generating a reduction of compression stresses for the shell with the opposite effect for the core (1). At the end of PCC shell material and interface core (38) are in compression while the centre of the roll is in tension due to the different values of CTE, the martensitic transformation or the very small thermal gradients through the roll.

Concerning phase composition, the core material contains a mixed structure Ferrite/Pearlite structure with a distribution that changes within the core thickness. In the centre of the roll (1) the phase amount is $65 \% \mathrm{Pe}-35 \% \mathrm{Fe}$ and in the core interface (38) it is $74 \% \mathrm{Pe}-26 \% \mathrm{Fe}$. On the other hand, the shell material exhibits the same amount of Austenite and Martensite that corresponds to $52 \% \mathrm{Au}-48 \% \mathrm{Ma}$.

Residual stresses and geometry sensitivity. From simulations performed, it is possible to obtain the stresses evolution along the roll radius at different times. Fig.7(a) provides the axial stress at several times during the THT. Results for the heating stage are presented in continuous lines while results for the cooling stage are presented with dashed lines. It is clearly noticed that for core material, the stresses during heating are decreasing reaching the lower stress level at 390hr; on the contrary for shell material at the same moment the highest stress level is reached. From $395 \mathrm{hr}$ the inverse effect starts to take place and almost the same stress state is recovered at the end of THT for $415 \mathrm{hr}$ as the end of the post cast cooling. However the stress state at the end of THT (415hr) is slightly lower than at the end of PCC or beginning of THT (280hr) for core and shell material and the entire roll may exhibit improved mechanical properties due to the presence of a tempered martensite in the shell material that is known have an enhanced toughness compared to the brittle untempered martensite obtained at the end of PCC.

Three different geometries were simulated in order to study the effect of a different size of cylinders. The three studied geometries can be set as follows with respect to the outer diameter of the roll and its shell thickness respectively: $1200-80 \mathrm{~mm}$ which is the reference geometry presented in the sections above, $1000-50 \mathrm{~mm}$ and $1300-150 \mathrm{~mm}$ as the additional cases for the comparison. From the industry, it has been observed than for diameter equal to $1000 \mathrm{~mm}$ no failure occurs, however for diameters of $1200 \mathrm{~mm}$ failure could occur and the failure rate increases for diameter $1300 \mathrm{~mm}$. Fig.7(b) compares the residual stresses along the radius for these three geometries at the end of THT. The higher tensile residual stresses at core material were obtained for the biggest geometry, on the contrary, for the smallest geometry, the lower residual stresses were obtained. However for the shell, the same level of compression stresses is obtained. At the end of PCC the same relation is observed between three geometries simulated. However the comparison for the whole stress history is more complex to analyse and shows extreme values non proportional to total radius.

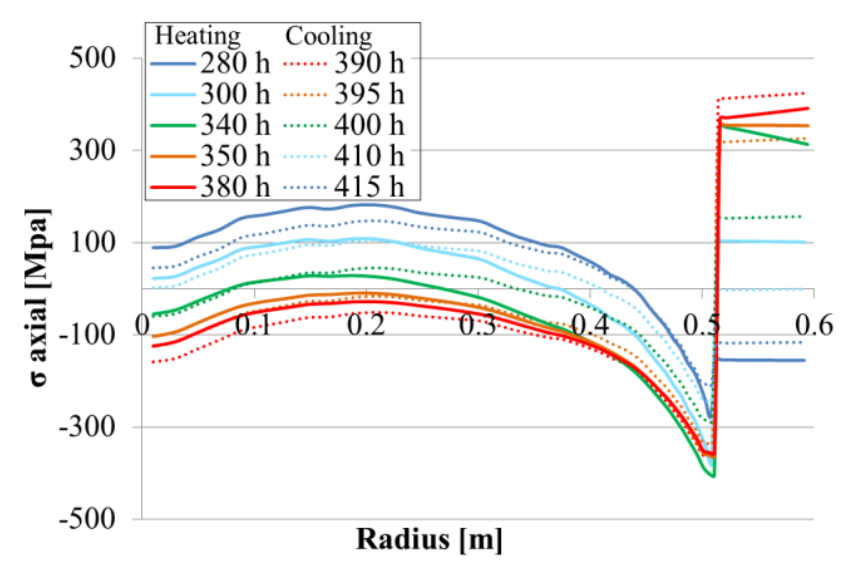

(a)

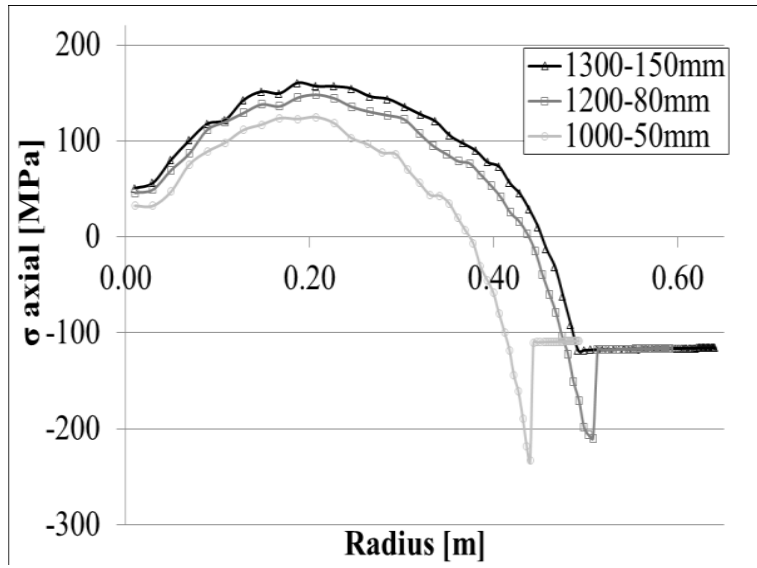

(b)

Fig.7: Axial stresses evolution (a) stress profiles along the roll radius during THT (b) comparison of residual stresses along the radius for three different geometries after THT. 


\section{Conclusion}

It was possible to obtain required parameters for simulations such as Coefficients of Thermal Expansion and Transformation Strain from experimental data. These parameters contribute to the characterization of studied materials and they strongly modify simulation results.

TTT diagrams for core and shell material were optimized by inverse method in order to obtain corresponding phase rates through numerical simulations.

From axial stress evolution obtained by simulations, it was possible to confirm the high stress level generated during the subsequent tempering heat treatment. This statement is in good agreement with the observations made in the actual industrial conditions as failure of the rolls often occurs during the heat treatment.

The performed simulations allow studying the interactions between mechanical and metallurgical features. During the cooling stage after casting process it was possible to clearly identify the influence of each phase transformation on the axial stresses evolution.

The current study leads to the enhancement of large stress gradients generated within the interface between the core and the shell materials during heat treatment, with the core material remaining in a compression state while the stress significantly increases in the shell material which exhibits a high tension state. For future work, a gradient of materials at the interface may be considered.

The comparison performed between three different roll geometries are in good agreement with the experimental observation on the failure issue on the rolls as they help confirming the increase of the failure rate with the outer diameter of the roll especially above a threshold value. So simulation predicted trends are validated even if residual stress measurements for current steel grades are lacking for the current rolls.

Acknowledgements. The authors acknowledge Conicyt (National commission for scientific and technological research) Chile, for financial help. Interuniversity Attraction Poles Program-Belgian State - Belgian Science Policy P7 INTEMATE is thanked for its support. As research Director of FRS-FNRS, AM Habraken thanks this fund, for financial support.

\section{References}

[1] A. M. Habraken and M. Bourdouxhe, "Coupled thermomechanical- metallurgical analysis during the cooling of steel pieces," Eur. J. Mech. - A/Solids, vol. 11, pp. 381-402, 1992.

[2] I. Neira Torres, G. Gilles, J. Tchoufang Tchuindjang, J. Lecomte-Beckers, M. Sinnaeve, and A. M. Habraken, "Prediction of residual stresses by FE simulations on bimetallic work rolls during cooling," Comput. Methods Mater. Sci., vol. 13, no. 1, pp. 84-91, 2013.

[3] I. Neira Torres, "Identificación de diagramas TTT a partir de diagramas CCT para modelación de transformaciones de fase," Final work, Universidad de Concepción, 2011.

[4] M. Carton and J. Lecomte-Beckers, "Rapport d'essais réalisés sur de matériaux de coeur," 2009.

[5] Q. Contrepois and J. Lecomte-Beckers, "Analyses thermo physiques de l'acier haut chrome," 2011.

[6] D. Koistinen and R. . Marburger, "A General Equation prescribing extent of the austenitemartensite transformation in pure Fe-C alloys and plain carbon steels," Acta Metall., vol. 7, pp. 59-60, 1959.

[7] W. A. Johnson and R. F. Mehl, Trans. AIME, p. 416, 1939. 